\title{
Anomalous refractive properties of photonic crystals
}

\author{
Boris Gralak, Stefan Enoch and Gérard Tayeb \\ Laboratoire d'Optique Électromagnétique, ESA 6079, Faculté des Sciences et Techniques, \\ Centre de Saint-Jérôme, 13397 Marseille Cedex 20, France
}

\begin{abstract}
:
We describe methods of investigation in order to study the behavior of photonic crystals. Our approach establishes a link between the dispersion relation of the Bloch modes for an infinite crystal (which describes the intrinsic properties of the photonic crystal in the absence of an incident field) and the diffraction problem of a grating (finite photonic crystal) illuminated by an incident field. We point out the relationship between the translation operator of the first problem, and the transfer matrix of the second. The eigenvalues of the transfer matrix contain information about the dispersion relation. This approach enables us to answer questions such as: when does ultrarefraction occur? Can the photonic crystal simulate a homogeneous and isotropic material with low effective index? This approach also enables us to determine suitable parameters to obtain ultrarefractive or negative refraction properties and to design optical devices such as highly dispersive microprisms and ultrarefractive microlenses. Rigorous computations add a quantitative aspect, and demonstrate the relevance of our approach.
\end{abstract}

\section{INTRODUCTION}

Photonic crystals have been the subject of considerable interest in the last decade. They have many potential technological applications such as the development of efficient semiconductor light emitters, filters, substrates for microwave antennas, lossless mirrors. Consequently, photonic crystals have generated intensive experimental and theoretical research.

Experimental studies of ultra refraction, negative refraction, highly dispersive properties of structures based on photonic crystals have been reported recently ${ }^{1,2}$. To our knowledge, the most thorough study has been performed on corrugated waveguides ${ }^{3}$. However, the electromagnetic foundations for explaining these phenomena have not been clarified. Our contribution clarifies these foundations, and allows us to demonstrate specific properties of photonic crystals.

In this paper, we are interested in finite size photonic crystals illuminated by an incident field. A priori, this problem is different from the study of Bloch modes propagating in an infinite crystal. Bloch theory only considers propagative modes, whereas in a finite crystal, there are also evanescent modes. It is well known in grating theory that evanescent modes play an important role and cannot be neglected in a quantitative analysis. In section 2, we recall some well-known results for Bloch waves in infinite crystals.

Section 3 is concerned with an actual problem of diffraction by finite size crystals. With the help of an elementary transfer matrix, we point out the connections between the incident field, the eigensolutions of this matrix, and the Bloch modes of the associated infinite structure.

In section 4, we show how these tools can be used to understand and anticipate the qualitative behavior of limited crystals, and we apply them to the study of anomalous refraction properties of photonic crystals. We give examples of ultrarefraction and negative refraction.

Sections 5 and 6 are devoted to devices using anomalous refraction properties: the qualitative rules given in the preceding section enable us to determine the parameters that permit the design of ultrarefractive microlenses and highly dispersive microprisms. Rigorous computations add a quantitative aspect, and demonstrate the relevance of our approach.

There are circumstances where evanescent modes govern the behavior of the crystal. The simplest example is obtained inside a bandgap, and we show in section 7 how field decay is related to the eigenvalues of the elementary transfer matrix.

Throughout the paper, we use a rectangular coordinate system $(O, x, y, z)$. The unit vectors of the axes are $\mathbf{e}_{x}, \mathbf{e}_{y}$ and $\mathbf{e}_{z}$. We consider harmonic fields represented using a time dependence $\exp (-i \omega t)$, with $\omega=2 \pi c / \lambda=c k_{0}, \quad c$ being the celerity of light in vacuum and $\lambda$ the wavelength. All the numeric values of the linear measurements given in the examples are in arbitrary units.

For the sake of simplicity, we consider in this paper twodimensional photonic crystals made with lossless materials (dielectric or perfectly conducting). However, the generalization to three-dimensional structures is straightforward. The reason for restricting ourselves to $2 \mathrm{D}$ cases is that vast computational means, not currently available, would be needed to rigorously solve realistic $3 \mathrm{D}$ problems. The $2 \mathrm{D}$ photonic crystal is invariant by translation along the $z$-axis. We suppose that the total electromagnetic field is $z$-independent. Consequently, the problem reduces to two independent scalar problems that we call $\mathrm{E} / /$ (resp. H//) when the electric (resp. magnetic) field is parallel to the $z$-axis. We denote by $u(x, y)$ the relevant component of the total field ( $E_{z}$ or $H_{z}$ depending on the polarization case).

\section{BLOCH SOLUTIONS IN AN INFINITE CRYSTAL}

Solutions of the Maxwell equations in an infinite periodic structure have been extensively studied 4-9. The permittivity $\varepsilon$ of the $2 \mathrm{D}$ problem is invariant under two fundamental and independent translations $\mathbf{d}$ and $\Delta$ :

$$
\text { for all integers } p \text { and } q, \quad \varepsilon(\mathbf{r}+p \mathbf{d}+q \Delta)=\varepsilon(\mathbf{r}) \text {. }
$$

The theoretical background is based on Bloch theorem: every solution is a linear combination of Bloch eigenmodes. The relevant field component of each Bloch eigenmode is such that:

$$
u_{\mathbf{k}}(\mathbf{r})=\exp (\mathrm{i} \mathbf{k} \cdot \mathbf{r}) v(\mathbf{r}),
$$

where $v(\mathbf{r})$ is a periodic function:

$$
\text { for all integers } p \text { and } q, \quad v(\mathbf{r}+p \mathbf{d}+q \Delta)=v(\mathbf{r}) .
$$

In the usual sense of the Bloch theorem, the Bloch wave vector $\mathbf{k}$ is real, since one is concerned with bounded solutions. This assumption also holds in the continuation of this section.

There are several methods to obtain the solutions $\mathbf{k}(\omega)^{4-9}$. From these solutions, the dispersion relationships can be derived. In the classical presentation, the solutions are presented on a bidimensional diagram where the abscissa represents the edge of the first reduced Brillouin zone. We give in figure 1 an example of this diagram in the case of a photonic crystal made of circular rods of radius $\rho=0.475$, with optical index $v=3$, lying in vacuum. The rods are arranged on a square lattice with period $d=1.27$. These parameters are those of the previous experimental and theoretical works depicted in ${ }^{10-12}$. Apart from section 7, all the photonic crystals used in this paper keep these parameters. Figure 1 has been computed using a plane wave expansion method ${ }^{5}$. It clearly shows the presence of several gaps. Many of the further examples of this paper will concern the upper limit of the second gap (ordered in increasing values of $\omega$ ). 


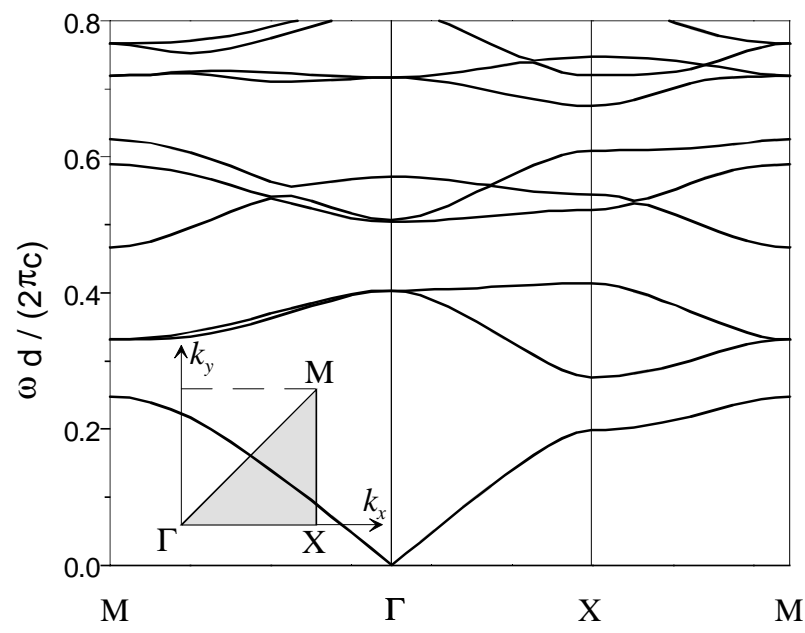

Figure 1: Dispersion diagram for E// polarization in a $2 D$ crystal with square lattice. The abscissa represents the Bloch wave vector on the edge of the first reduced Brillouin zone shown in the small insert: $\Gamma, X$ and $M$ stand for the points with coordinates $(0,0),(\pi / d, 0)$ and $(\pi / d, \pi / d)$ in the $\left(k_{x}, k_{y}\right)$ plane.

In order to study the transport of energy inside the crystal, let us recall a fundamental result linking the dispersion relation and the energy propagation ${ }^{13}$. For a given Bloch wave, we denote by $\mathbf{V}_{e}$ the average velocity of the energy flow (the average is taken upon a lattice cell):

$$
\mathbf{V}_{e}=\frac{\iint_{\text {cell }}(\text { Poynting vector }) \mathrm{d} \mathbf{r}}{\iint_{\text {cell }}(\text { energy density }) \mathrm{d} \mathbf{r}},
$$

and by $\mathbf{V}_{g}$ the group velocity of the same Bloch wave, deduced from the dispersion relation $\omega(\mathbf{k})$ by:

$$
\mathbf{V}_{g}=\operatorname{grad}_{\mathbf{k}}(\omega)=\frac{\partial \omega}{\partial k_{x}} \mathbf{e}_{x}+\frac{\partial \omega}{\partial k_{y}} \mathbf{e}_{y}
$$

The result is that

$$
\mathbf{V}_{e}=\mathbf{V}_{g} \text {. }
$$

All these results are valid for structures that fill the entire space. In the next section, we show the relationship between the external incident field of a physical problem and these Bloch waves.

\section{T-MATRIX OF A PHOTONIC CRYSTAL SLICE}

In this section, we make use of the T-matrix that represents the translation operator between the top and the bottom sides of a grating (a photonic crystal slice). Its eigensolutions are a valuable tool in order to understand the crystal behavior. This point of view has already been suggested in the computation of band structures ${ }^{14,15}$. We show that they also give information on transmission properties and on the coupling between the incident field and the Bloch waves.

\subsection{The crystal as a grating problem; pseudo-periodicity of the fields}

The crystal is modeled as a stack of $N$ grids (Fig. 2). Each grid is equivalent to a grating. As well known in grating theory, the Tmatrix that relates the field above and below a grating suffers from the exponential growth of its elements. Consequently, the use of the T-matrix leads to numerical problems, at least when the structure is composed of several grids. Many other propagation algorithms are more suitable from this point of view ${ }^{16-20}$. As regards our objectives, we will make use of the T- matrix related to only one grid, in such a way that the numerical problems do not arise. On the other hand, the T-matrix contains all the information on the propagation of the field through the structure, and makes a simple connection between gratings problems ( $N$ is a finite number) and Bloch waves in infinite crystals ( $N$ infinite).

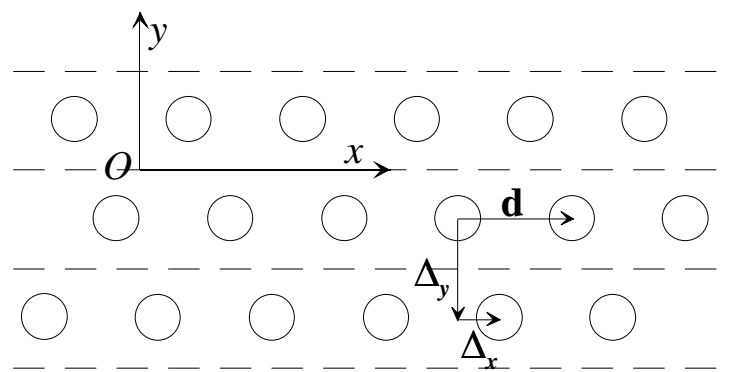

Figure 2: A crystal with finite extent with respect to the $y$-direction, and made of $N$ gratings ( $N=3$ on this example). The structure is z-independent, and infinite along the $x$-direction. The periodicity along $x$ is $d$. The distance between two grids is $\Delta_{y}$. The $x$-shift between two grids is $\Delta_{x}$. Thus, the two elementary translations are $\mathbf{d}=d \mathbf{e}_{x}$ and $\Delta=\Delta_{x} \mathbf{e}_{x}-\Delta_{y} \mathbf{e}_{y}$. Each grating is characterized by $x$-periodic electromagnetic parameters, which are not necessarily piecewise constant.

In a general problem, this grating is surrounded by an electromagnetic field with no particular properties (no radiation wave condition, no plane wave expansion). For instance, we can think of a problem where this grating is a slice of a thicker photonic crystal, illuminated by a limited beam. The relevant field component can be written as a Fourier integral:

$$
u(x, y)=\int_{-\infty}^{+\infty} \hat{u}(\alpha, y) \exp (\mathrm{i} \alpha x) \mathrm{d} \alpha .
$$

By splitting the integration interval $]-\infty,+\infty[$ in subintervals $\left[n \frac{2 \pi}{d},(n+1) \frac{2 \pi}{d}\right]$, a simple change of variable leads to the other expression:

$$
u(x, y)=\int_{0}^{2 \pi / d} u_{\alpha}(x, y) \mathrm{d} \alpha,
$$

where the integrand

$$
u_{\alpha}(x, y)=\sum_{m=-\infty}^{-\infty} \hat{u}\left(\alpha+m \frac{2 \pi}{d}, y\right) \exp \left(\mathrm{i}\left(\alpha+m \frac{2 \pi}{d}\right) x\right)
$$

is a $x$-pseudo-periodic function ${ }^{21}$ with pseudo-periodicity coefficient $\alpha$, i.e.:

$$
u_{\alpha}(x+d, y)=\exp (\mathrm{i} \alpha d) u_{\alpha}(x, y) .
$$

Consequently, the study of the general field $u(x, y)$ reduces to the study of its pseudo-periodic components $u_{\alpha}(x, y)$ for all $\alpha$ in the first Brillouin zone $[0,2 \pi / d]$ of the $x$-periodic problem.

\subsection{Definition of the T-matrix}

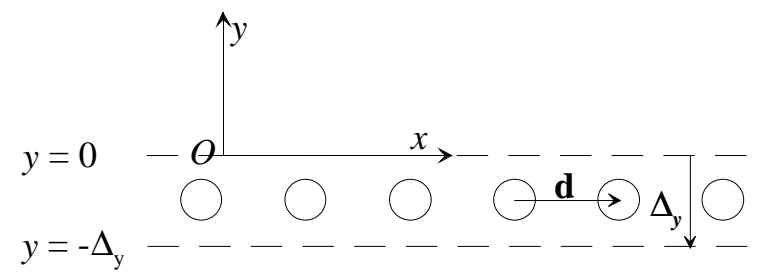

Figure 3: A single grating extracted from figure 2.

Let us isolate a grating from the complete stack (Fig. 3). The medium above and below this grating is homogeneous and can be arbitrarily chosen. Without loss of generality, we assume that this 
medium is the vacuum. As well known in grating theory, the pseudo-periodic function $u_{\alpha}(x, y)$ is written as:

$$
\begin{gathered}
\text { for } y>0: \\
\begin{array}{c}
u_{\alpha}(x, y)=\sum_{m=-\infty}^{+\infty}\left[A_{m}^{-} \exp \left(-\mathrm{i} \beta_{m} y\right)+A_{m}^{+} \exp \left(+\mathrm{i} \beta_{m} y\right)\right] \exp \left(\mathrm{i} \alpha_{m} x\right),(1 \\
\text { for } y<-\Delta_{y}: \\
u_{\alpha}(x, y)=\sum_{m=-\infty}^{+\infty}\left[B_{m}^{-} \exp \left(-\mathrm{i} \beta_{m}\left(y+\Delta_{y}\right)\right)+B_{m}^{+} \exp \left(+\mathrm{i} \beta_{m}\left(y+\Delta_{y}\right)\right)\right] \\
\times \exp \left(\mathrm{i} \alpha_{m}\left(x-\Delta_{x}\right)\right)
\end{array}
\end{gathered}
$$

where

$$
\alpha_{m}=\alpha+m \frac{2 \pi}{d}
$$

and $\beta_{m}$ is defined by:

$$
\alpha_{m}{ }^{2}+\beta_{m}{ }^{2}=\frac{\omega^{2}}{c^{2}}, \text { with } \arg \left(\beta_{m}\right) \in\{0, \pi / 2\} .
$$

Denoting by $A^{-}, A^{+}, B^{-}$and $B^{+}$the infinite column vectors with $A_{m}^{-}, A_{m}^{+}, B_{m}^{-}$and $B_{m}^{+}$components, the T-matrix is the operator which relates the field below the grating to the field above:

$$
\left[\begin{array}{l}
B^{-} \\
B^{+}
\end{array}\right]=T\left[\begin{array}{l}
A^{-} \\
A^{+}
\end{array}\right]=\left[\begin{array}{ll}
T_{11} & T_{12} \\
T_{21} & T_{22}
\end{array}\right]\left[\begin{array}{l}
A^{-} \\
A^{+}
\end{array}\right] .
$$

The T-matrix is obtained using any good numerical technique able to solve the problem of figure 3 , illuminated by an appropriate plane wave (which is associated to the required value of $\alpha$ in $[0,2 \pi / d]$. In our implementation, we use a numerical code based on a rigorous integral theory ${ }^{22}$. This code is one of the basic tools in our laboratory for the study of gratings and nondoped photonic crystals. It is the latest development of the original thesis work of D. Maystre ${ }^{23}$, and has been thoroughly checked by classic tests and comparisons with experimental data. With the help of this code, we compute first the S-matrix defined by

$$
\left[\begin{array}{l}
A^{+} \\
B^{-}
\end{array}\right]=\left[\begin{array}{ll}
S_{11} & S_{12} \\
S_{21} & S_{22}
\end{array}\right]\left[\begin{array}{l}
A^{-} \\
B^{+}
\end{array}\right] .
$$

From the computed S-matrix (necessarily of finite size), we derive a finite size approximate of the T-matrix by the classical relations (which can be easily obtained from (15) and (16)):

$$
\left\{\begin{array}{l}
T_{11}=S_{21}-S_{22} S_{12}^{-1} S_{11} \\
T_{12}=S_{22} S_{12}{ }^{-1} \\
T_{21}=-S_{12}{ }^{-1} S_{11} \\
T_{22}=S_{12}{ }^{-1}
\end{array} .\right.
$$

The resulting finite size transfer matrix will be denoted by $T_{M}, M$ being defined in such a way that the summations in (11) and (12) go from $-M$ to $+M$.

\subsection{Eigenvalues of the T-matrix}

The T-matrix represents the translation operator between the top and the bottom sides of the grating. Let us consider one eigenvalue $\mu$ of the transfer matrix $T_{M}$, and let us call $A_{\mu}$ the associated eigenvector. The components of this eigenvector can be regarded as $A_{\mu, m}^{-}$and $A_{\mu, m}^{+}$coefficients of a field $u_{\alpha, \mu}(x, y)$ defined by (11) in the upper region $(y>0)$ :

$$
A_{\mu}=\left[\begin{array}{c}
A_{\mu}^{-} \\
A_{\mu}^{+}
\end{array}\right] \text {. }
$$

Note that the field $u_{\alpha, \mu}(x, y)$ associated to the eigenvector $A_{\mu}$ is defined everywhere, and in particular in the grating region $\left(-\Delta_{y} \leq y \leq 0\right)$. The coefficients $B_{\mu, m}^{-}$and $B_{\mu, m}^{+}$of this field are defined by (12) below the grating $\left(y<-\Delta_{y}\right)$ and verify:

$$
B_{\mu}=\left[\begin{array}{c}
B_{\mu}^{-} \\
B_{\mu}^{+}
\end{array}\right]=T_{M}\left[\begin{array}{c}
A_{\mu}^{-} \\
A_{\mu}^{+}
\end{array}\right]=T_{M} A_{\mu}=\mu A_{\mu}=\mu\left[\begin{array}{c}
A_{\mu}^{-} \\
A_{\mu}^{+}
\end{array}\right] .
$$

From (11) and (12), it emerges that the values of the field $u_{\alpha, \mu}$ at the points with coordinates $(x, 0)$ and $\left(x+\Delta_{x}, y-\Delta_{y}\right)$ only differ by a multiplicative coefficient $\mu$.

Let us suppose that $|\mu|=1$. Then the multiplicative coefficient is a pure phase shift. It means that the restriction of $u_{\alpha, \mu}$ in the slice $\left(-\Delta_{y} \leq y \leq 0\right)$ can also be considered as the restriction in the same region of the infinite $2 \mathrm{D}$ periodic problem of section 2 . In other words, this eigenvector represents in the slice a Bloch solution $u_{\mathbf{k}}(\mathbf{r})$. The fields $u_{\alpha, \mu}$ and $u_{\mathbf{k}}$ respectively verify Eqs. (10) and (2):

$$
\begin{aligned}
& u_{\alpha, \mu}(\mathbf{r}+\mathbf{d})=\exp (\mathrm{i} \alpha d) u_{\alpha, \mu}(\mathbf{r}), \\
& u_{\mathbf{k}}(\mathbf{r}+\mathbf{d})=\exp \left(\mathrm{i} k_{x} d\right) u_{\mathbf{k}}(\mathbf{r}),
\end{aligned}
$$

which gives the $x$-component of the Bloch vector:

$$
k_{x}=\alpha \text {. }
$$

Applying the second elementary translation $\Delta$ :

$$
\begin{aligned}
& u_{\alpha, \mu}(\mathbf{r}+\Delta)=\exp (\mathrm{i} \arg (\mu)) u_{\alpha, \mu}(\mathbf{r}), \\
& u_{\mathbf{k}}(\mathbf{r}+\Delta)=\exp \left(\mathrm{i} k_{x} \Delta_{x}-\mathrm{i} k_{y} \Delta_{y}\right) u_{\mathbf{k}}(\mathbf{r}),
\end{aligned}
$$

give the $y$-component of the Bloch vector:

$$
k_{y}=\frac{k_{x} \Delta_{x}-\arg (\mu)}{\Delta_{y}} .
$$

Equations (22) and (25) point out the link between the grating problem and the Bloch solutions of the infinite structure. Moreover, equation (25) paves the way for obtaining the dispersion curves of the Bloch problem from the eigenvalues of the T-matrix.

Let us suppose that $|\mu| \neq 1$. The restriction to the slice $\left(-\Delta_{y} \leq y \leq 0\right)$ of $u_{\alpha, \mu}$ associated to the eigenvector can not be a Bloch solution with real vector $\mathbf{k}$.

Let us now consider the spectrum of the transfer matrix $T_{M}$. We can distinguish the two following situations.

First, if there is no eigenvalue with modulus equal to 1 , it could appear evident that we are in a "gap" situation: if the crystal of figure 2 is illuminated by an incident plane wave (with a wave vector projection on the $x$-axis equal to $\alpha$ ), the transmitted field through the $N$ gratings tends exponentially towards zero as $N$ increases. This exponential decay is directly related to the modulus of the eigenvalue whose modulus is the closest to 1 . An example will be given in section 7. In order to prove this assumption, one should consider the translation operator $\mathrm{T}$ (infinite matrix). Indeed, the truncated matrix $T_{M}$ has lost some fundamental properties of $\mathrm{T}$ (such as those linked to the energy conservation), and therefore it is difficult to quantify the pertinence of $\left(T_{M}\right)^{N}$ when $N$ tends to infinity. This problem involves subtle mathematics and the interested reader will find mathematical background in a recent paper ${ }^{24}$.

Second, if there is at least one eigenvalue with modulus equal to 1 , there is no gap in the sense of the $2 \mathrm{D}$ crystal that fills the entire space. In other words, there is at least one Bloch solution that can propagate in the structure. If the crystal of figure 2 is illuminated by a plane wave, the incident field can excite the Bloch solution. 
But the coupling between the incident field and the Bloch solution also depends on numerous parameters, and in particular on the respective symmetries of these two fields ${ }^{25-28}$.

In any case, the field in the grating can never be reduced to a combination of Bloch waves. The methods that rely upon this assumption can probably give accurate results in some circumstances, but their results should be carefully checked with the help of rigorous methods. The same applies to conclusions directly obtained from dispersion diagrams of Bloch waves. However, as will be shown in the next sections of the paper, these dispersion diagrams are very helpful in the prediction and the understanding of the complex phenomena that occur in photonic crystal studies.

\section{USEFULNESS OF THESE TOOLS}

\subsection{Three-dimensional dispersion diagram}

From now on, we suppose that the photonic crystal has a square lattice. Therefore, the elementary translations are such that $\Delta_{x}=0$ and $\Delta_{y}=d$. All the examples are given in the $\mathrm{E} / /$ polarization case.

Even though most of the information about propagating waves (i.e. $|\mu|=1$ ) can be deduced from bidimensional dispersion diagrams as that shown in figure 1 , we found that a 3D representation of the same diagram was more convenient for our purpose. Figure 4 shows the same dispersion diagram as figure 1, but the Bloch wave vector covers the whole Brillouin zone (in fact the square region where $k_{x}$ and $k_{y}$ belong to $[0, \pi / d]$ ) instead of the edge of the first reduced Brillouin zone. In this figure, each band is represented by a sheet. This diagram reveals the complexity of the dispersion relation of the photonic band structure, and especially how the three higher sheets of this figure overlap at the upper limit of the second gap.

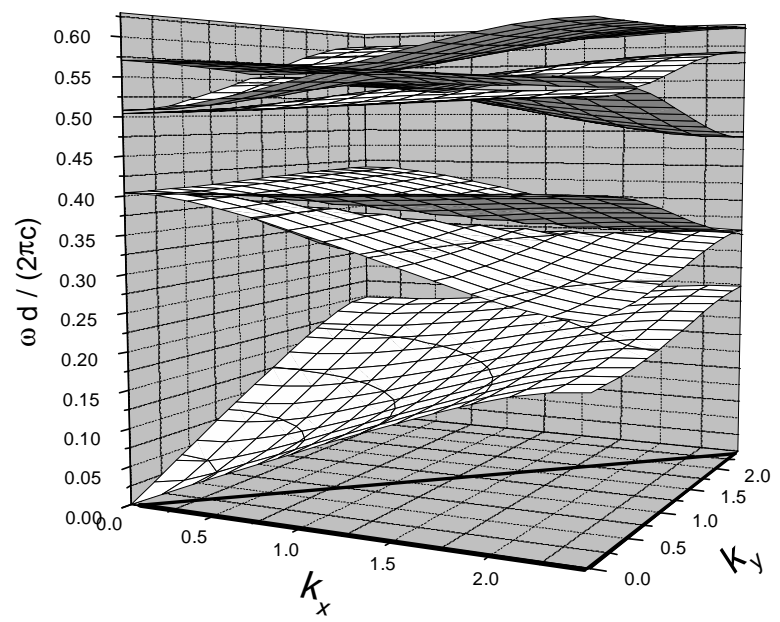

Figure 4: Three-dimensional dispersion diagram. The horizontal plane gives the Bloch wave vector $\mathbf{k}$. The vertical axis gives the normalized frequency $\omega d /(2 \pi c)=d / \lambda$. The bottom sides of the sheets are represented in darker gray. The triangle corresponding to the first reduced Brillouin zone has been drawn in the $\left(k_{x}, k_{y}\right)$ plane. The parameters are the same as in figure 1 . The diagram has been computed using the plane wave expansion method.

Since several interesting properties of the photonic crystals are obtained at the limit of the gap, let us plot (Fig. 5) an enlarged view of this region.

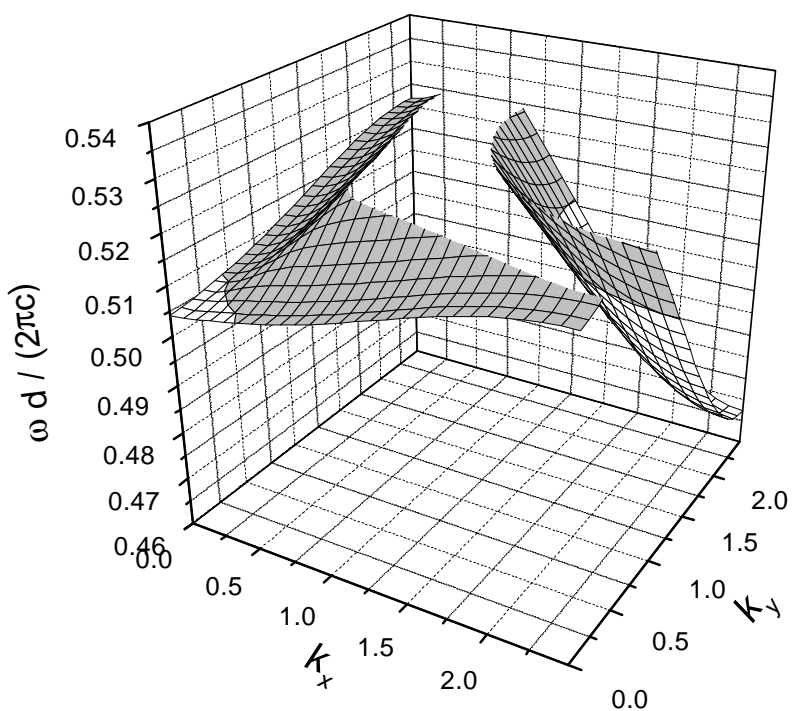

Figure 5: Enlarged view of the 3D-dispersion diagram near the upper edge of the second gap. The intersection between the sheets and the horizontal plane corresponding to the wavelength $\lambda=2.545$ is the curve that limits the two differently colored regions of the sheets. Due to the slow convergence of the plane wave expansion method, the levels of the sheets are not perfectly accurate, and this diagram only gives qualitative information.

The problems we are concerned with are harmonic ones. Hence we are interested in the intersection of the sheets and the horizontal plane associated to the actual value of $\omega$. This intersection is pointed out (for an arbitrary chosen $\omega$ ) by the limit between the two differently colored regions of the sheets. The resulting curves are reported on Fig. 6 . Note that the wavelength $\lambda=2.545$ in Figs. 5 and 6 is chosen in order to match the previous work of reference ${ }^{12}$.

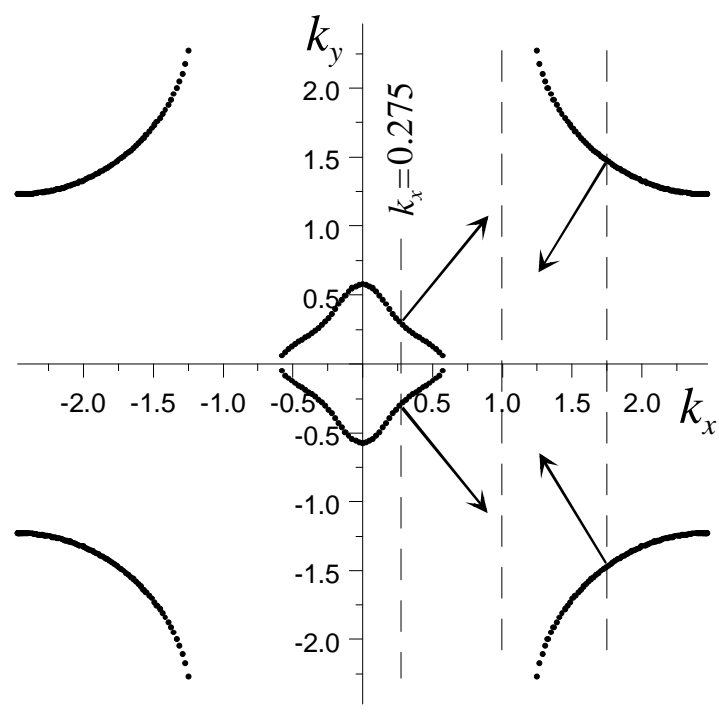

Figure 6: Constant-frequency dispersion diagram and energy flow direction for some particular values of $k_{x}$. The dotted curves are the intersection of the sheets (Fig. 5) and the horizontal plane corresponding to $\lambda=2.545$. There is no propagating solution for $0.6<k_{x}<1.2$. This quantitative diagram is obtained using the T-matrix. 


\subsection{Constant-frequency dispersion diagram}

It is worth noting that the plane wave expansion method is well suited to obtain the Bloch solutions for a given value of the Bloch wave vector $\mathbf{k}$, and is adapted to the plot of dispersions diagrams such as that presented in figure 1 . But it suffers from a poor convergence rate versus the number of plane waves. In our problem, and since we are at the edge of the gap, a very small error in the dispersion diagram can drastically change the conclusions.

On the other hand, the use of the T-matrix directly gives the solutions $k_{y}$ as a function of $k_{x}$ (Eqs. (22) and (25)) for a given value of the wavelength $\lambda$. Moreover, this method gives a very good accuracy and can deal with any grating structure, including dispersive and metallic materials.

That is the reason why figure 6 has been computed by the search of the eigenvalues (with $|\mu|=1$ ) of the $T_{M}$ matrix. In this case, $M=7$ is sufficient to ensure a good accuracy. The graph shows that in this particular case, for each fixed value of $k_{x}$ in [$\pi / d, \pi / d]$, there are zero or two opposite values of $k_{y}$ in $[-\pi / d, \pi / d]$. Remark that due to the symmetry of this crystal, if $k_{y}$ is solution, $-k_{y}$ is also a solution.

Let us now focus on the propagation of the energy for a given Bloch wave. Equations (5) and (6) tell us that the averaged velocity of the energy flow is immediately given by the normal to the constant-frequency dispersion diagram (Fig. 6). Moreover, the combined use of (5) and figure 5 allows us to determine the orientation of this vector, which points towards the increasing values of $\omega$ on the sheet of Fig. 5.

\subsection{Application to a finite crystal}

Let us now consider a finite crystal with $N$ grating layers, illuminated by an incident field. We suppose in a first step that this incident field is a plane wave with wavevector of modulus $k^{i}$ and incidence $\theta$ with respect to the $y$-axis. From grating theory, it is well known that the pseudo-periodicity coefficient $\alpha$ (Eq. (10)) is given by:

$$
\alpha=k^{i} \sin \theta \text {. }
$$

In order to make a prediction about the field behavior in the illuminated crystal, we only consider the propagating eigenvectors (with $|\mu|=1$ ) of the $T_{M}$ matrix which, from section 3.3, are associated with Bloch solutions of the infinite crystal. One should be aware of the empirical aspect of this hypothesis which neglects the evanescent solutions $(|\mu| \neq 1)$. One knows that similar assumptions in grating theory can lead to erroneous results. Nevertheless, we will see that in this way we obtain an interesting description of the energy travel in the crystal, which proves to be in good agreement with our rigorous numerical checks. From equation (22), we know that the pseudo-periodicity coefficient $\alpha$ is nothing more than the $x$-component of the Bloch wave vector, which gives the link between the incident plane wave and the Bloch solutions.

In a second step, let us suppose that the incident field is a limited beam expressed as a plane wave packet:

$$
u^{i}(x, y)=\int_{-\infty}^{+\infty} A(\alpha) \exp (\mathrm{i} \alpha x-\mathrm{i} \beta(\alpha) y) \mathrm{d} \alpha,
$$

with $\alpha=k^{i} \sin \theta, \beta^{2}=k^{i^{2}}-\alpha^{2}$, and with a gaussian amplitude:

$$
A(\alpha)=\frac{W}{2 \sqrt{\pi}} \exp \left(-\frac{\left(\alpha-\alpha_{0}\right)^{2} W^{2}}{4}\right) .
$$

The mean incidence $\theta_{0}$ of the beam is such that:

$$
\alpha_{0}=k^{i} \sin \theta_{0} \text {. }
$$

It can be noticed that the parameter $W$ appearing in (28) is directly linked to the incident beam width.
There is no major difference with the case of an incident plane wave, and each component $A(\alpha) \exp (\mathrm{i} \alpha x-\mathrm{i} \beta(\alpha) y)$ of the plane wave packet has an average energy flow direction given by the remark of section 4.2, i.e. the normal to the constant-frequency dispersion diagram at the points given by $k_{x}=\alpha$.

\subsection{Anomalous beam shift and ultrarefractive behavior}

Coming back to figure 6 , we can profit from the quasi-square shape of the central curve: we illuminate the crystal by a limited beam with $\alpha_{0}=0.275$. This value corresponds in vacuum to a mean incidence $\theta_{0}=6.4^{\circ}$ for $\lambda=2.545$, which are the parameters already used in Ref. ${ }^{12}$. For a value $W=10$ of the beam width, the amplitude $A(\alpha)$ take significant values (namely $A(\alpha)>A\left(\alpha_{0}\right) / 5$ ) only for $0.02<\alpha<0.53$. All the directions normal to the constant-frequency dispersion diagram are close to the $\pm 45^{\circ}$ directions, depending whether the energy flows upwards or downwards.

These parameters are those used for the computation of figure 7 . This total field map has been computed with the help of a modal method based on scattering matrices, the fields being expressed as Fourier Bessel series ${ }^{29,27}$. This method rigorously solves the problem of a finite set of parallel rods. From this point of view, the structure is not a grating as considered in previous sections (infinite extent along the $x$-axis). Anyway, in our case where the incident field is a limited beam, there is no noticeable difference between the fields diffracted by the finite set of rods and by the grating, at least when (as can be seen on Fig. 7) the field vanishes at both ends of the crystal. A comparison between the $x$-truncated crystal and the grating is given in Ref. ${ }^{12}$. This modal method has been chosen because it enables us to deal also with structures which can not be modeled by gratings: microlenses (section 5) or microprisms (section 6).

Figure 7 shows that our expectations concerning the average energy flow inside the crystal are confirmed by the rigorous computation. Roughly, the problem looks like a light ray going through a slice of material with optical index less than 1 . Following the Snell-Descartes law, the transmitted beam is abnormally shifted with respect to the axis of the incident beam. Both upward and downward directions can be interpreted by the reflection of the beam. This example has already been studied in Ref. ${ }^{12}$. But the approach is here completely different, and probably more intuitive.

It is worth noting that in this particular case, and since the central curve of figure 6 is not an ellipse (and a fortiori not a circle) we can not consider the photonic crystal as a homogeneous material with low effective permittivity tensor (and a fortiori with low effective optical index). Nevertheless, this example clearly exhibits an ultrarefractive behavior. 


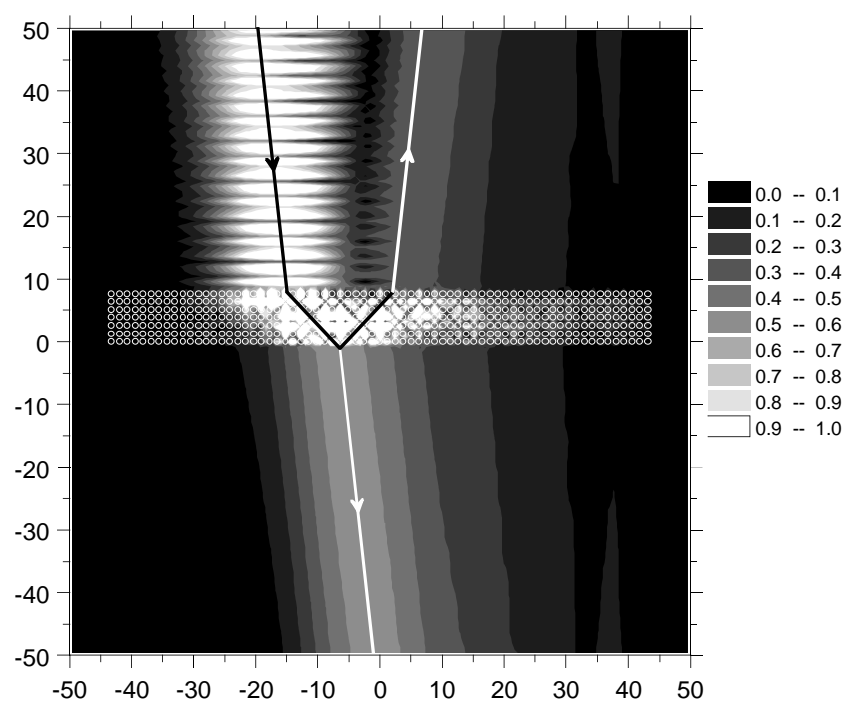

Figure 7: Map of the total field modulus for a crystal made of $69 \times 7$ rods lying in vacuum and illuminated by an E// polarized gaussian beam with $\theta_{0}=6.4^{\circ}$. The crystal parameters are the

same as in Fig. 1. Above the crystal the beam reflected by the crystal interferes with the incident beam and generates a system of stationary waves. Straight lines show the locus of the maximum incident (black), transmitted and reflected (white) fields.

\subsection{Negative refraction}

Figure 6 shows that for large positive values of $k_{x}$, the average energy propagates towards the negative values of $x$. This phenomenon can be checked by increasing the mean incidence to a value $\theta_{0}=40^{\circ}$. The corresponding field map is shown in Fig. 8. A ray interpretation for this particular incidence will lead to a negative refraction. Note that in order to consider the material as a homogeneous one with negative optical index, the constant-frequency dispersion diagram should look like a circle centered on the $\Gamma$ point $\left(k_{x}=k_{y}=0\right)$ on a sheet that reaches its maximum at $\Gamma$. A similar situation in a $3 \mathrm{D}$ crystal has been recently reported by Kosaka et al. ${ }^{2}$.

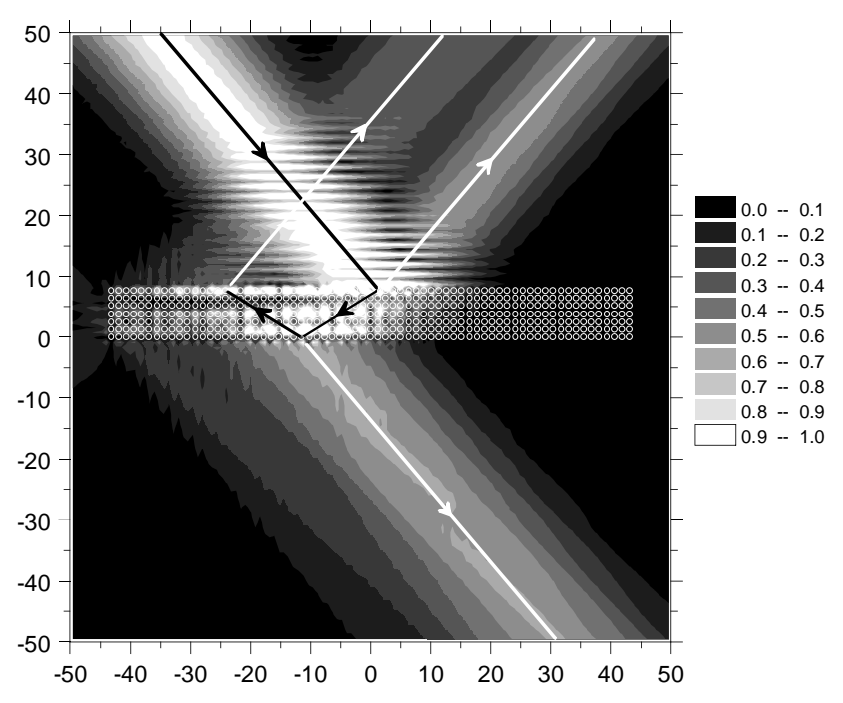

Figure 8: Negative refraction. Same parameters as Fig. 7, except $\theta_{0}=40^{\circ}$

\section{APPLICATION TO ULTRAREFRACTIVE MICROLENSES}

Ultrarefractive properties of photonic crystals have been suggested previously ${ }^{30}$. We show here that the formalism exposed in the previous sections is well suited to determine the parameters that enable to engineer devices based on this phenomenon. The aim is to design an artificial material whose effective index is less than 1 , and if possible close to zero. It means that the constant-frequency dispersion diagram should follow a circle

$$
k_{x}^{2}+k_{y}^{2}=k_{0}^{2} n_{e f f}^{2},
$$

i.e. a small circle centered on the origin in the $\left(k_{x}, k_{y}\right)$ plane. In order to obtain this property, let us decrease the frequency associated to the horizontal intersection plane of Fig. 5. Figure 9 shows the constant-frequency dispersion diagram for two wavelengths close to the $k_{x}=k_{y}=0$ limit of the sheet of Fig. 5 . The curve for $\lambda_{1}=2.56$ is very close to a circle, and the effective index given by (30) is $n_{1}=0.086$.

Note that even if the crystal behaves as a homogeneous material, the physical situation is very different from that generally studied in homogenization works where one considers quasi-static limits ${ }^{31,32}$.

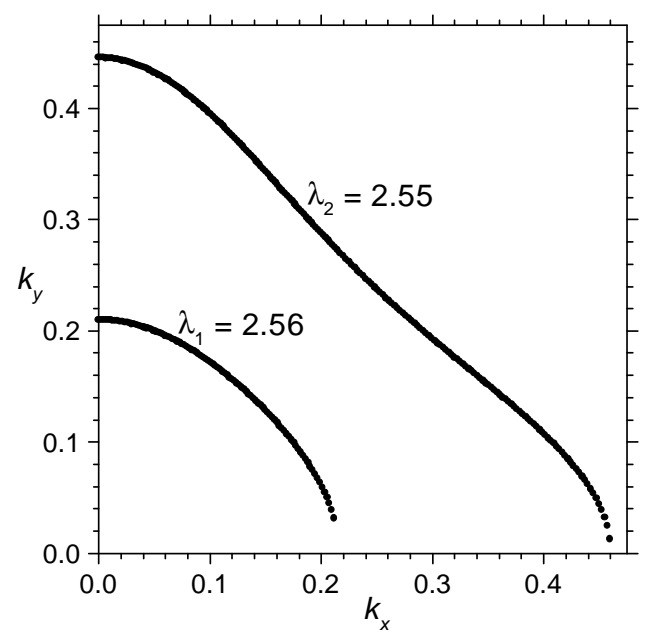

Figure 9: Same as Fig. 6 for two different wavelengths. Only the central region is presented.

Since the index contrast with other materials is very large, new optical elements can be imagined. For instance, let us design a microlens composed of 295 rods of the same photonic crystal. The width of this lens is equal to 64 , i.e. $25 \lambda$, and the radius of curvature of the concave face is $R=50$. When illuminated by a gaussian beam of width $W=40$ in normal incidence, the transmitted field focuses at a point situated at $f=53$ from the concave face (Fig. 10). Note that the same lens built in classical material (optical index greater than 1) is divergent. On the sides of Fig. 10, the field is principally due to the diffraction by the edges of the lens.

A straight application of optical geometric results gives the focal length:

$$
f=\frac{1}{1-n_{1}} R=54.7
$$

which agrees with the actual value $f=53$, and proves the very good similarity between the crystal and an isotropic homogeneous material.

One should be aware of the fact that if the constant-frequency dispersion diagram does not look like a circle, there is no clear focusing of the light. 


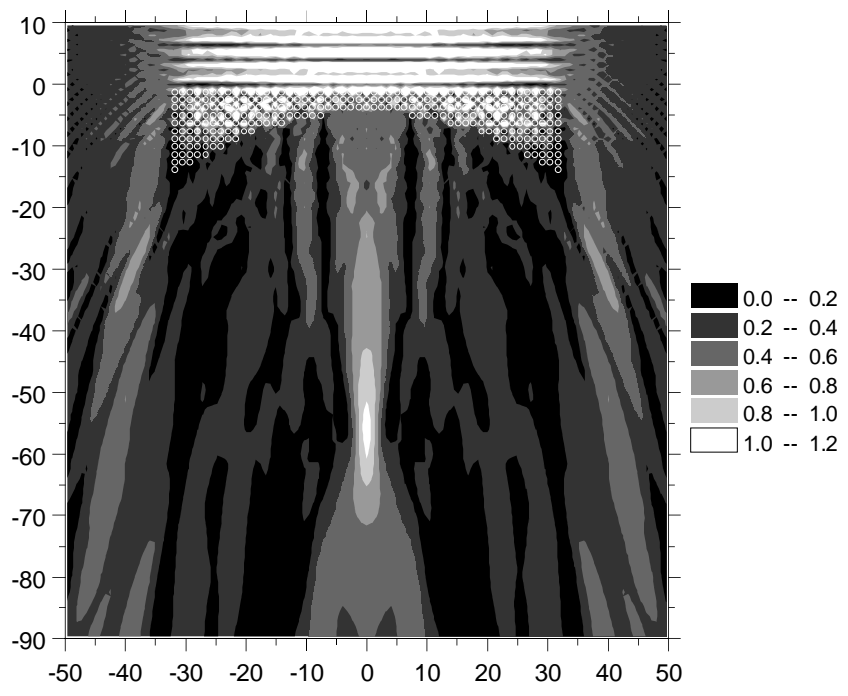

Figure 10: The microlens is illuminated in normal incidence from the top by a gaussian beam in E// polarization.

\section{APPLICATION TO HIGHLY DISPERSIVE MICROPRISMS}

Photonic crystal prisms have been experimentally studied and proposed as highly dispersive components ${ }^{1}$.

As a first step, let us work in the range of wavelengths where the crystal behaves as a quasi-homogeneous material. We chose the wavelength $\lambda_{1}=2.56$ for which the central curve of the constantfrequency dispersion diagram looks like a circle (Fig. 9). The effective optical index obtained from (30) is $n_{1}=0.086$. We consider a microprism made of 465 rods disposed in an isosceles right-angled triangle. The side of this triangle is equal to 37 , i.e. $15 \lambda$. It is illuminated by a gaussian beam of width $W=10$ in normal incidence. Figure 11 shows that the beam is going through the hypotenuse with quasi-normal direction. In order to precise this direction, we plot (Fig. 12) the scattered intensity at infinity versus the diffraction angle $\theta$ (conventional polar angle with horizontal axis as origin). A straight application of the SnellDescartes law leads to the following angle $\theta_{1}$ at the wavelength $\lambda_{1}$ :

$$
n_{1} \sin 45^{\circ}=\sin \left(45^{\circ}-\theta_{1}\right) \Rightarrow \theta_{1}=41.5^{\circ} .
$$

This value is in perfect agreement with Fig. 12.

We can remark that the high index contrast implies that the reflection is important at each interface. It could probably be attenuated by the use of some antireflection structure.

In a second step, let us evaluate the dispersion. Changing the wavelength to $\lambda_{2}=2.55$ shifts the maximum diffraction angle

(Fig. 12) by about $3^{\circ}$. By the way, since this wavelength is farther from the gap, the transmitted intensity is greater. Figure 9 shows that for this wavelength, the constant-frequency dispersion diagram is not a circle, but it has no importance on the phenomenon. Indeed, and since this problem only involves "plane boundaries" between the crystal and the external vacuum, the crystal does not necessarily need to behave as a homogeneous material.

It is easy to verify that the dispersion $d \theta / d \lambda$ is much greater with this microprism than with any other classical dispersive device (grating, silica prism). Such microprisms could find interesting applications in the domain of fiber optical communications, and in particular in wavelength multiplexing/demultiplexing.

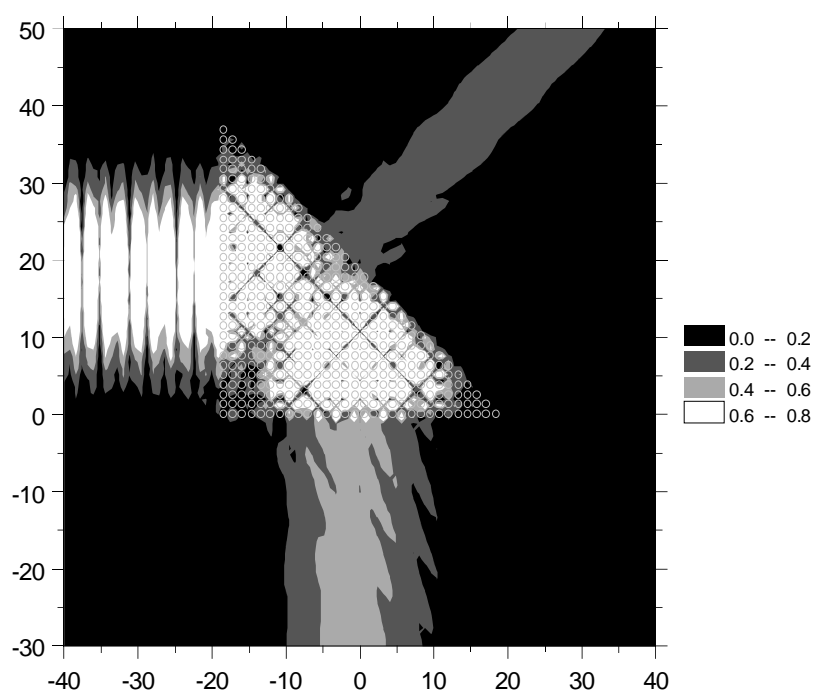

Figure 11: The microprism is illuminated from the left by a gaussian beam with $\lambda_{1}=2.56$.

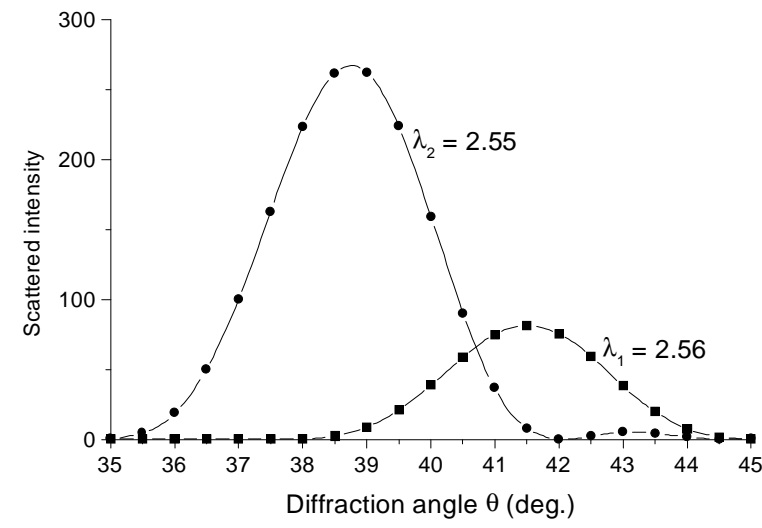

Figure 12: Scattered intensity at infinity for $\lambda_{1}=2.56$ and $\lambda_{2}=2.55$.

\section{NON PROPAGATIVE SOLUTIONS AND T-MATRIX FORMALISM}

In the previous examples, our interpretation was based on Bloch solutions, and the evanescent solutions were neglected. We have shown that this point of view leads to many interesting predictions in the transparency bands. When there is no Bloch solution (gap), one can extract from the eigenvalues of the Tmatrix some pertinent information.

The global behavior of the field inside the crystal is governed by the eigenvectors of the T-matrix. We consider a semi-infinite crystal, i.e. $N$ tends to infinity in Fig. 2, and the crystal fills the space for $y$ less than an arbitrary value.

Inside the gap, there is no eigenvalue with modulus equal to 1 . All the eigenvectors are evanescent or anti-evanescent with respect to $y$. Since the field should stay bounded when $y$ tends towards $-\infty$, only the eigenvectors associated with the eigenvalues with modulus less than 1 are suitable. From all of them, the one whose eigenvalue $\mu_{1}$ is the closest to 1 has the slowest decay. From this remark, for a sufficient number $N$ of layers, we can expect that the energy transmission behaves as $\left|\mu_{1}\right|^{2 N}$ (the energy is linked to the square of the eigenvector).

We illustrate this property in the case of a photonic crystal made of $N$ layers of perfectly conducting rods. The radius of the rods is equal to 0.01 . The square cell of the crystal has a spacing $d=1$, and the crystal is illuminated in normal incidence by a plane wave in $E / /$ polarization, at a wavelength $\lambda=10.2$. These data are those of a previous paper ${ }^{33}$. From the T-matrix of a single layer, 
we obtain $\left|\mu_{1}\right|=0.288$. Figure 13 compares the decimal logarithm of the transmission (obtained with a rigorous grating code) through a stack of $N$ grating layers and the decimal logarithm of $\left|\mu_{1}\right|^{2 N}$. The slopes are identical; thus the exponential decay is clearly governed by this eigenvalue. This spectacular agreement is due to the fact that in this case the moduli of the other eigenvalues are considerably smaller than $\left|\mu_{1}\right|$. Otherwise, one should probably consider a greater number of layers in order to see the predominance of $\mu_{1}$.

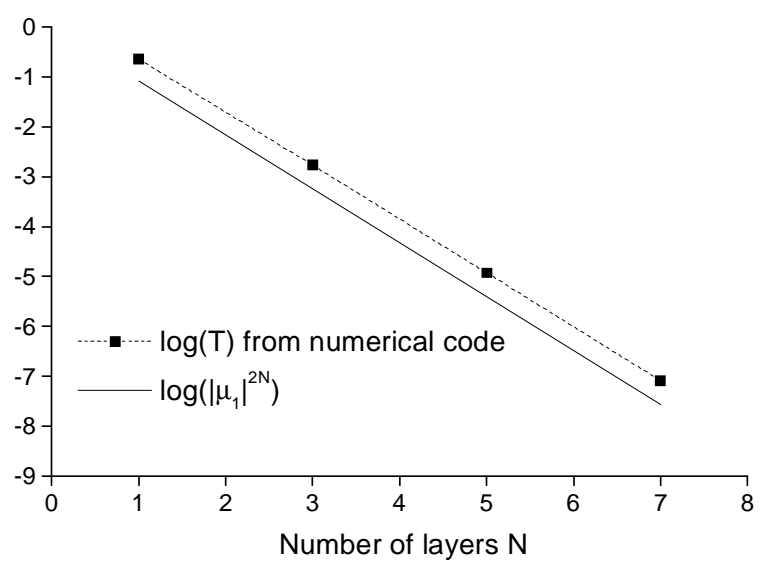

Figure 13: Exponential decay of the transmission for a photonic crystal made of $N$ layers of perfectly conducting rods.

\section{Conclusion}

We have developed an efficient approach in order to predict some of the properties specific to photonic crystals. The theoretical basis has been exposed. Dispersion diagrams of Bloch modes are useful in this context. However, the Bloch theory does not take into account the fact that the crystal is of limited extent, illuminated by an incident field, and it neglects evanescent waves that exist in physical situations. That is why our final step includes a careful check with the help of rigorous numerical codes. It also gives information on the coupling between the incident field and the structure, which strongly depends on the symmetries. Moreover, it is the only way to obtain the energy transported by the different beams.

From a practical point of view, the way to find the right parameters giving rise to anomalous refraction situations has been pointed out. In some circumstances depicted in section 5 , the photonic crystal can simulate a homogeneous and isotropic material with low effective index. Note that a good understanding of photonic crystals properties is necessary in order to find these right parameters, and we must confess that our first attempts to design a microlens without the help of the present paper considerations have been unsuccessful.

We have pointed out some phenomena which are not only spectacular, but also have potential practical developments. Due to recent technological advances, applications involving components such as highly dispersive microprisms and ultrarefractive microlenses should appear soon.

\section{$\underline{\text { References }}$}

[1] S.Y. Lin, V.M Hietala, L. Wang, E.D. Jones, "Highly dispersive photonic band-gap prism", Opt. Lett. 21, 17711773 (1996)

[2] H. Kosaka, T. Kawashima, A. Tomita, M. Notomi, T. Tamamura, T. Sato and S. Kawakami, "Superprism phenomena in photonic crystals", Phys. Rev. B 58, 1009610099 (1998)

[3] R. Zengerle, "Light propagation in singly and doubly periodic planar waveguides", J. Mod. Opt. 34, 1589-1617, (1987)
[4] K.M. Ho, C.T. Chan and C.M. Soukoulis, "Existence of photonic gap in periodic dielectric structures", Phys. Rev. Lett. 65, 3152-3155 (1990)

[5] M. Plihal and A.A. Maradudin, "Photonic band structure of two-dimensional systems: the triangular lattice", Phys. Rev. B 44, 8565-8571 (1991)

[6] H.S. Sözüer, J.W. Haus and R. Inguva, "Photonic bands: convergence problems with the plane-wave method", Phys. Rev. B 45, 13962-13972 (1992)

[7] J. Joannopoulos, R. Meade, and J. Winn, Photonic crystals, Princeton University Press, 1995

[8] R.D. Meade, A.M. Rappe, K.D. Brommer, J.D. Joannopoulos, O.L. Alerhand, "Accurate theoretical analysis of photonic band-gap materials", Phys. Rev. B 48, 8434-8437 (1993). See also the erratum in Phys. Rev. B 55, 15942 (1997)

[9] A. Moroz and C. Sommers, "Photonic band gaps of threedimensional face-centered cubic lattices", J. Phys.: Condens. Matter 11, 997-1008 (1999)

[10] D.R. Smith, S. Schultz, S.L. McCall, P.M. Platzmann, "Defect studies in a two-dimensional periodic photonic lattice", J. Mod. Opt. 41, 395-404 (1994)

[11] M. Sigalas, C.M. Soukoulis, E.N. Economou, C.T. Chan, and K.M. Ho, "Photonic band gaps and defects in two dimensions: studies of the transmission coefficient", Phys. Rev. B 48, 14121-14126 (1993)

[12] S. Enoch, G. Tayeb, D. Maystre, "Numerical evidence of ultrarefractive optics in photonic crystals", Optics Comm. 161, 171-176 (1999)

[13] P. Yeh, "Electromagnetic propagation in birefringent layered media", J. Opt. Soc. Am. 69, p.742-756, 1979

[14] J.B. Pendry, "Photonic band structures", J. Mod. Opt. 41, 209-229 (1994)

[15] J.M. Elson and P. Tran, "Coupled-mode calculation with the R-matrix propagator for the dispersion of surface waves on a truncated photonic crystal", Phys. Rev. B 54, 17111715 (1996)

[16] R.C. Hall, R. Mittra, and K.M. Mitzner, "Analysis of multilayered periodic structures using generalized scattering matrix theory", IEEE Trans. Ant. Prop. 36, 511517 (1988)

[17] M. Nevière, F. Montiel, "Deep gratings: a combination of the differential theory and the multiple reflection series", Optics Comm. 108, 1-7 (1994)

[18] F. Montiel and M. Nevière, "Differential theory of gratings: extension to deep gratings of arbitrary profile and permittivity through the R-matrix propagation algorithm", J. Opt. Soc. Am. A 11, 3241-3250 (1994)

[19] L. Li, "Bremmer series, R-matrix propagation algorithm, and numerical modeling of diffraction gratings, J. Opt. Soc. Am. A 11, 2829-2836 (1994)

[20] L. Li, "Formulation and comparison of two recursive matrix algorithms for modeling layered diffraction gratings", J. Opt. Soc. Am. A 13, 1024-1035 (1996)

[21] Electromagnetic Theory of gratings, Ed. R. Petit, SpringerVerlag, Berlin, 1980

[22] D. Maystre, "Electromagnetic study of photonic band gaps", Pure Appl. Opt. 3, 975-993 (1994)

[23] D. Maystre, "Sur la diffraction et l'absorption par les réseaux utilisés dans l'infrarouge, le visible et l'ultraviolet; applications à la spectroscopie et au filtrage des ondes électromagnétiques", thesis, Université Aix-Marseille 3, Marseille, 1974

[24] L. Li, "Justification of matrix truncation in the modal methods of diffraction gratings", J. Opt. A: Pure Appl. Opt. 1, 531-536 (1999)

[25] P. Villeneuve, S. Fan, J.D. Joannopoulos, "Microcavities in photonic crystals: mode symmetry, tunability, and coupling efficiency", Phys. Rev. B 54, 7837-7842 (1996) 
[26] A. Sentenac, J.J. Greffet and F. Pincemin, "Structure of the electromagnetic field in a slab of photonic crystal", J. Opt. Soc. Am. B 14, 339-347 (1997)

[27] G. Tayeb, D. Maystre, "Rigorous theoretical study of finite size two-dimensional photonic crystals doped by microcavities", J. Opt. Soc. Am. A 14, 3323-3332 (1997)

[28] Z. Yuan, J.W. Haus, K. Sakoda, "Eigenmode symmetry for simple cubic lattices and the transmission spectra", Optics Express 3, 19-27 (1998)

[29] D. Felbacq, G. Tayeb, and D. Maystre, "Scattering by a random set of parallel cylinders", J. Opt. Soc. Am. A 11, 2526-2538 (1994)

[30] J.P Dowling and C.M. Bowden, "Anomalous index of refraction in photonic bandgap materials", J. Mod. Opt. 41, 345-351 (1994)

[31] R.C. McPhedran, N.A. Nicorovici, L.C. Botten, "The TEM mode and homogenization of doubly periodic structures", J. Electrom. Waves and Appl. 11, 981-1012 (1997)

[32] D. Felbacq, G. Bouchitté, "Homogenization of a set of parallel fibers", Waves in Random Media 7, 245-256 (1997)

[33] G. Guida, D. Maystre, G. Tayeb, P. Vincent, "Mean-field theory of two-dimensional metallic photonic crystals", J. Opt. Soc. Am. B 15, 2308-2315 (1998) 